\title{
Is Cannabis of Potential Value as a Therapeutic for Inflammatory Bowel Disease?
}

\author{
Timna Naftali ${ }^{1,2}$
}

Published online: 6 August 2019

(c) Springer Science+Business Media, LLC, part of Springer Nature 2019

Cannabis is commonly used by patients with inflammatory bowel disease (IBD) to ameliorate their symptoms. Patients claim that cannabis reduces pain, increases appetite, and reduces the need for other medications [1-3]. The prevalence of cannabis use in patients with IBD is $15-40 \%$ [4], including among pediatric patients [5]. Nevertheless, few scientifically sound data exist regarding the value of cannabis in the treatment of IBD. This discrepancy between the patient's perception of cannabis as an effective medicine and physician's reluctance to use non-evidence-based treatment often creates a conflict between patients and physicians, with few IBD patients consulting their medical doctors about cannabis use [6]. More evidence about the value of cannabis as a treatment for inflammatory bowel disease is urgently needed.

Although the cannabis plant contains over 100 different cannabinoids [7], research has focused mainly on $\Delta$ 9-tetrahydrocannabinol (THC) and cannabidiol (CBD). THC is the substance responsible for the psychoactive effects of cannabis, including euphoria, pain relief, and memory impairment [8], whereas CBD has an anti-inflammatory effect [9] as well as a protective effect against THCinduced psychosis [10]. Other substances contained in the plant include other cannabinoids, terpenes, and flavonoids that act synergistically and are responsible for strain-specific differences [11, 12].

The phytocannabinoids activate the cannabinoid receptors CB1, CB2, TRPV1, and GPR55 [13], all of which are part of the endocannabinoid system, a system that has an important homeostatic function including modulation of neuroprotection, pain, emotional memory, hunger, feeding,

Timna Naftali

timna.naftali@clalit.org.il

1 Institute of Gastroenterology and Hepatology, Meir Hospital Sapir Medical Center, Kfar Saba, Israel

2 The Sackler School of Medicine, Tel Aviv University, Tel Aviv, Israel lipid metabolism, and most importantly in the context of IBD, immunity, and inflammation [14, 15]. Many laboratory studies demonstrated that the endocannabinoid system is an important component of inflammatory activity in IBD and that treatment with cannabinoids can ameliorate the disease impact in experimental models of IBD by reducing inflammation and tissue damage $[4,16]$.

Human studies of cannabis in IBD are mostly limited to reports of the prevalence and description of the reasons for cannabis use among IBD patients. In a recent study by Pi et al., patients with IBD reported more cannabis use compared with the non-IBD population, $74.4 \%$ (125/168) versus $48.3 \%(28 / 58)(p<0.001)$. The cannabis users stated that cannabis improved their pain, appetite, and diarrhea [17]. Weiss et al. analyzed patterns of cannabis use among $2,084,895$ subjects with IBD and 2,013,901 control subjects. Subjects with IBD had a higher incidence of ever having used cannabis, started using at a younger age, and used a greater amount per day [18]. Storr et al. observed that cannabis had been used by $17.6 \%$ of respondents to their questionnaire and that in patients with Crohn's disease, the use of cannabis for $>6$ months was a strong predictor of surgical treatment (odds ratio $=5.03 ; 95 \%$ confidence inter$\mathrm{val}=1.45-17.46$ ) [19]. Contrary to that finding, Mbachi et al. compared the prevalence of Crohn's disease-related complications among patients who were cannabis users ( $n=615)$ and nonusers $(n=42,702)$ in a study published in this issue of Digestive Diseases and Sciences. They observed that cannabis users were less likely to develop Crohn's disease-related complications including active fistulizing disease, intra-abdominal abscess, need for blood transfusion, colectomy, and need for parenteral nutrition. Comorbidity was significantly lower among cannabis users, but the prevalence of concurrent psychiatric diseases was significantly higher [20]. The conflicting data between these two studies emphasize the question as to whether the beneficial effect of cannabis is the result of masking disease symptoms, or due to a true anti-inflammatory effect. 
The above-mentioned studies are all limited to inspecting the prevalence of cannabis use among IBD patients. Unfortunately, there are no data on the dose, type, and mode of cannabis consumption. Stith et al. used mobile device software to collect data from 3341 cannabis users affected with several diseases [21]. The main outcome was the change in symptom severity, as reported by the patient. THC levels were independently associated with greater symptom relief, whereas CBD levels were not, implying that THC is the substance responsible for the symptomatic improvement reported by the patients. Nonetheless, conclusions regarding the appropriate dose of cannabis in these diseases cannot be drawn from that study.

Randomized controlled studies investigating cannabis use in IBD are few and small. We performed a preliminary double-blind, placebo-controlled study of cannabis use in Crohn's disease in which 21 patients were given cigarettes containing $11.5 \mathrm{mg}$ of THC or placebo [22]. A decrease in Crohn's disease activity index (CDAI) of $>100$ was observed in 10/11 (90\%) subjects in the cannabis group but only $4 / 10(40 \%)$ in the placebo group $(p=0.028)$. Yet, we did not observe changes in the inflammatory biomarker C-RP. A similar study using oral $10 \mathrm{mg}$ CBD twice daily in Crohn's disease was negative. Only 19 patients completed the study, and after 8 weeks of treatment, the CDAI was $220 \pm 122$ and $216 \pm 121$ in the CBD and placebo groups, respectively ( $p=$ NS) [23]. Irving et al., in a double-blind placebo-controlled study, assessed the efficacy of CBD-rich botanical extract, also containing $4 \%$ THC, in 60 ulcerative colitis patients [24]. Remission rates were similar for CBD (28\%) and placebo (26\%). The study drug was poorly tolerated, with only $59 \%$ protocol compliance, so when performing a per-protocol analysis of the partial and the total Mayo scores, the investigators found a significant difference in favor of the study drug $(p=0.068$ and $p=0.038$, respectively).

In conclusion, considering the mechanism of action of phytocannabinoids and the accumulating evidence of their anti-inflammatory effects in experimental and in vitro studies, it is reasonable to assume that cannabis can be of benefit in the treatment of IBD. While many of our patients are convinced that cannabis is ameliorating their disease manifestations, clinicians continue to observe rather than to investigate this phenomenon. If cannabis really has therapeutic potential, it must be studied further such as in the study by Mbachi et al. that points to a possibility of cannabis improving not only patients' symptoms but also disease outcome, a possibility that has to be further explored.

\section{References}

1. García-Planella E, Marín L, Domènech E, et al. Use of complementary and alternative medicine and drug abuse in patients with inflammatory bowel disease. Med Clin (Barc). 2007;128:45-48. (in Spanish).

2. Lal S, Prasad N, Ryan M, et al. Cannabis use amongst patients with inflammatory bowel disease. Eur J Gastroenterol Hepatol. 2011;23:891-896.

3. RavikoffAllegretti J, Courtwright A, Lucci M, Korzenik JR, Levine J. Marijuana use patterns among patients with inflammatory bowel disease. Inflamm Bowel Dis. 2013;19:2809-2814.

4. Ahmed Waseem, Katz Seymour. Therapeutic use of cannabis in inflammatory bowel disease. Gastroenterol Hepatol (N Y). 2016;12:668-679.

5. Hoffenberg EJ, Newman H, Collins C, Tarbell S, Leinwand K. Cannabis and pediatric inflammatory bowel disease: change blossoms a mile high. J Pediatr Gastroenterol Nutr. 2017;64:265-271.

6. Kerlin A, Long M, Kappelman M, Martin C, Sandler R. Profiles of patients who use marijuana for inflammatory bowel disease. Dig Dis Sci. 2018;63:1600-1604. https://doi.org/10.1007/s1062 0-018-5040-5.

7. Hanus LO. Pharmacological and therapeutic secrets of plant and brain (endo)cannabinoids. Med Res Rev. 2009;29:213-271.

8. Morrison PD, Zois V, McKeown DA, et al. The acute effects of synthetic intravenous Delta9-tetrahydrocannabinol on psychosis, mood and cognitive functioning. Psychol Med. 2009;39:1607-1616.

9. Burstein S. Cannabidiol (CBD) and its analogs: a review of their effects on inflammation. Bioorg Med Chem. 2015;23:1377-1385.

10. Morgan CJA, Schafer G, Freeman TP, et al. Impact of cannabidiol on the acute memory and psychotomimetic effects of smoked cannabis: naturalistic study: naturalistic study [corrected]. $\mathrm{Br} \mathrm{J}$ Psychiatry J Mental Sci. 2010;197:285-290.

11. Russo EB, Jiang HE, Li X, et al. Phytochemical and genetic analyses of ancient cannabis from Central Asia. J Exp Bot. 2008;59:4171-4182.

12. Russo EB. Taming THC: potential cannabis synergy and phytocannabinoid-terpenoid entourage effects. Br J Pharmacol. 2011;163:1344-1364.

13. Howlett AC, Barth F, Bonner TI, et al. International Union of Pharmacology. XXVII. Classification of cannabinoid receptors. Pharmacol Rev. 2002;54:161-202.

14. Turcotte C, Chouinard F, Lefebvre JS, Flamand N. Regulation of inflammation by cannabinoids, the endocannabinoids 2-arachidonoyl-glycerol and arachidonoyl-ethanolamide, and their metabolites. J Leukoc Biol. 2015;97:1049-1070.

15. McPartland JM, Guy GW, Di Marzo V. Care and feeding of the endocannabinoid system: a systematic review of potential clinical interventions that upregulate the endocannabinoid system. PLOS ONE. 2014;9:e89566.

16. Leinwand KL, Gerich ME, Hoffenberg EJ, Collins CB. Manipulation of the endocannabinoid system in colitis: a comprehensive review. Inflamm Bowel Dis. 2017;23:192-199.

17. Pi S, Rosenfeld G, Enns R, et al. Patterns and motivations of cannabis use amongst patients with inflammatory bowel disease. GastroHep. 2019;1:100-107.

18. Weiss A, Friedenberg F. Patterns of cannabis use in patients with inflammatory bowel disease: a population based analysis. Drug Alcohol Depend. 2015;1:6-11.

19. Storr M, Devlin S, Kaplan GG, Panaccione RAC. Cannabis use provides symptom relief in patients with inflammatory bowel disease but is associated with worse disease prognosis in patients with Crohn's disease. Inflamm Bowel Dis. 2014;20:472-480. 
20. Mbachi C, Attar B, Wang Y, et al. Association between cannabis use and complications related to Crohn's disease: a retrospective cohort study. Dig Dis Sci. (Epub ahead of print). https://doi. org/10.1007/s10620-019-05556-z.

21. Stith SS, Vigil JM, Brockelman F, Keeling K, Hall B. The association between cannabis product characteristics and symptom relief. Sci Rep. 2019;9:2712.

22. Naftali T, Bar-Lev Schleider L, Dotan I, Lansky EP, SklerovskyBenjaminov F, Konikoff FM. Cannabis induces a clinical response in patients with Crohn's disease: a prospective placebo-controlled study. Clin Gastroenterol Hepatol. 2013;11:1276-1280.e1. https ://doi.org/10.1016/j.cgh.2013.04.034.

23. Naftali T, Mechulam R, Marii A, et al. Low-dose cannabidiol is safe but not effective in the treatment for Crohn's disease, a randomized controlled trial. Dig Dis Sci. 2017;62:1615-1620. https://doi.org/10.1007/s10620-017-4540-z.

24. Irving PM, Iqbal T, Nwokolo C, et al. A randomized, doubleblind, placebo-controlled, parallel-group, pilot study of cannabidiol-rich botanical extract in the symptomatic treatment of ulcerative colitis. Inflamm Bowel Dis. 2018;24:714-724.

Publisher's Note Springer Nature remains neutral with regard to jurisdictional claims in published maps and institutional affiliations. 